DOI: $10.15593 / 2224-9354 / 2019.1 .4$

УДК 378.016

\author{
Н.А. Молодчик, Н.И. Нагибина
}

\author{
ФОРМИРОВАНИЕ И ВНЕДРЕНИЕ МОДЕЛИ \\ ПРАКТИКО-ОРИЕНТИРОВАННОГО ОБУЧЕНИЯ \\ В УНИВЕРСИТЕТЕ НА ОСНОВЕ AGILE-ПРИНЦИПОВ
}

\begin{abstract}
Социально-экономические и политические вызовы глобального мира вносят коррективы в современные потребности рынка труда: необходимость выпуска новых инновационных продуктов определяет потребность в ускорении получения специалистов с актуальными компетенциями с фокусом на когнитивные навыки вместо «загрузки» знаний. В условиях оптимизации государственных образовательных учреждений для большинства российских университетов своевременная трансформация является единственным способом выжить. Гипотезой исследования является применимость agile-концепции в сфере высшего образования в России: использование agileпринципов в управлении структурными подразделениями университета может оказать значительное положительное влияние на повышение эффективности образовательного процесса и рост конкурентоспособности вуза. Предложенная модель практико-ориентированного обучения, основанная на принципах agile, заимствованная из сферы информационных технологий, подтвердила актуальность в практике предприятий различных сфер деятельности.

Модель, основанная на концепции Agile - нового культурного феномена, образа мышления, философии, трансформирует формат и характер взаимодействия «университет-студентработодатель» непосредственно в процессе обучения: каждый из субъектов является активным элементом системы и в любой момент времени может стать инициатором каких-либо предложений или изменений. В модели формально выделено 5 основных блоков: а) процесс обучения; б) преподаватели кафедры; в) кураторы предприятий; г) востребованный выпускник; д) мотивированный абитуриент. Результаты апробации модели в управлении структурным подразделением университета - кафедрой менеджмента и маркетинга Пермского национального исследовательского политехнического университета выражены положительной динамикой изменения трех классических ключевых эффектов: повышением качества, ускорением процессов и снижением затрат.

Ключевые слова: модель практико-ориентированного обучения, agile-принципы, университет, внедрение.
\end{abstract}

Введение. V.U.C.A., по мнению J. Sullivan ${ }^{1}$, характеризующаяся нестабильностью, неопределенностью, сложностью и неясностью, определяет тренды сегодняшнего времени, в том числе и в России. Цифровизация экономики и общества накладывает серьезные отпечатки на изменяющиеся потребности работодателей и, как следствие, изменяет рынок труда. У предприятий сокращается время ожидания «прибытия» новых прогрессивных инно-

(С) Молодчик Н.А., Нагибина Н.И., 2019

Молодчик Наталья Анатольевна - канд. экон. наук, доцент кафедры менеджмента и маркетинга ФГБОУ ВО «Пермский национальный исследовательский политехнический университет», e-mail: namdom@yandex.ru.

Нагибина Наталья Ивановна - канд. экон. наук, доцент кафедры менеджмента и маркетинга ФГБОУ ВО «Пермский национальный исследовательский политехнический университет», e-mail: nagibinan@list.ru.

${ }^{1}$ Sullivan J. Доклад на Международном саммите HR - Digital 2016 (Москва, 15-16.09.2016). 
вационно развитых выпускников - специалистов. При этом сегодня в России университеты не удовлетворяют потребности работодателей в полной мере, $91 \%$ работодателей отмечают нехватку практических знаний у выпускников [1]. Этими трендами обусловлена актуальность поиска новых форматов взаимодействия университета и работодателя, а также новых механизмов и моделей управления образовательным процессом.

Agile: теоретический обзор и практики организаций. Изучение теоретических основ концепции Agile и опыта ее внедрения в различных организациях сформировало основу для реализации разработанной авторами модели.

A. De Smet, W. Aghina [2] рассматривают Agile в аспекте организационной гибкости и интерпретируют как проворный, быстрый, верткий, ловкий, маневренный. D.K. Rigby, J. Sutherland, H. Takeuchi [3] называют Agile адаптивной моделью. Анализируя три закона agile-организации: закон малой команды, закон клиента и закон сети, S. Denning [4] аргументированно доказывает, что: «Мы движемся к миру, где... Agile будет просто управлять, и обычным способом управлять компанией будет Agile». Полностью соглашаясь с представленными выше характеристиками, мы также разделяем позицию участников Agile Alliance [5], определяющих Agile как способность создавать и реагировать на изменения, чтобы преуспеть в неопределенной и бурной среде.

Изучив концептуальные основы Agile, мы можем предположить, что в целях получения готового продукта (в том числе в сфере образования) в условиях поликомандности, сокращения сроков и снижения стоимости возможно применение двух подходов:

1. Отождествляя Agile с прилагательным «гибкий», называя новым стилем управления, способом организационной деятельности, набором инструментов, методикой гибкого подхода к управлению, семейством гибких методологий [6], применять отдельнье составляющие Agile или применять Agile при реализации отдельных проектов и задач [7].

2. Определяя Agile как новый культурный феномен, образ мышления, философию, в центре которой «...не организация, а человечность: увеличение роли индивидуального потребителя и сотрудников организации...» $[8,9$, 20], когда «люди и взаимодействие важнее (над) процессами и инструментами» [10], применять концеепцию Agile в самом широком его смысле.

Практика последних 15 лет демонстрирует выход Agile за рамки разработки программного обеспечения: Кремниевая долина, Facebook, Amazon, Google и Yandex, Uber, Intel, Dell, Tesco и другие гиганты воплощают подходы Agile [11-14].

Самым ярким подтверждением использования agile-подхода в траектории развития системы российского образования является концепция «Университет 3.0». Полная реализация концепции, разработанной по инициативе Агентства стратегических инициатив РФ и находящейся на стадии поэтапно- 
го проектного внедрения, на всей территории России планируется к 2020 году. Основные элементы «Университета 3.0»: цифровая платформа; образовательный инжиниринг; база данных образовательных активностей (в том числе нейротехнологии); новые механизмы финансирования [15]. Интересным и полезным представляется опыт применения Agile в образовательной среде зарубежных стран. EduScrum [16] - специальный фонд, созданный при поддержке делового сообщества Нидерландов (пионеры agile-подхода в школьном образовании), помогает педагогам и учащимся изучить гибкую методологию Scrum и применять ее в процессе школьного обучения. Уже и в России начинается использование Agile в проектной деятельности детей [17]. В системе управления образованием Agile внедрен в университетах Cornell University и Northern Arizona University.

Таким образом, многообразие реализации agile-подхода в практике коммерческих и государственных предприятий подтверждает актуальность его дальнейшего развития.

Формирование и внедрение модели практико-ориентированного обучения в университете на основе agile-принципов.

1. Концепция формирования модели практико-ориентированного обучения на основе agile-принципов. В соответствии со стратегией и программами развития ПНИПУ в 2016 году на кафедре менеджмента и маркетинга было принято решение об актуализации подходов к построению образовательного процесса, отвечающим вызовам современности [19].

В основу нового подхода были положены следующие принципы Agile:

- Модель с ориентацией на подход инновационного переосмысления создания продукта. Подразумевая под продуктом студента, мы создали модель, в которой стремимся перманентно удерживать в фокусе инновационное переосмысление траекторий и технологий развития студента.

- Фокусировка на новой роли заказчика: заказчик как участник разработки продукта. В нашем случае заказчиком выступает как сам студент, так и работодатель. В новой модели образовательного процесса оба эти заказчика являются активными участниками agile-взаимодействия в команде.

- Фокусировка на результате - готовом работающем продукте, полностью соответствующем потребностям заказчика. С целью максимального приближения нашего продукта, т.е. студента, к потребностям заказчика мы фокусируемся не только на конечном результате - выпускнике, но и на промежуточных результатах. Студенты каждого курса, начиная с первого, это наш промежуточный результат.

- Уникальность при организации работь над созданием продукта: a) развитие подавляющего большинства студентов строится на индивидуальном гибком подходе как со стороны работодателя, так и со стороны кафедры; б) минимизируются работы, связанные с оформлением документов, подтвер- 
ждающих пребывание студента на предприятии, что экономит время и снижает барьеры доступа в организации; в) форматы сотрудничества с работодателями, учебные курсы находятся в постоянном совершенствовании с конечным результатом.

- Командно-клиентоориентированный подход: «эмочиональный» («дружный») коллектив - самоорганизующаяся кросс-функциональная команда. Модель коллаборации «кафедра-студент-работодатель», построенная на эмпатии и психологической безопасности, позволяет достичь хорошего синергетического эффекта.

- Гибкие методологии: открытые образовательные ресурсы на учебном портале кафедры, регулярно обновляемые форматы конференций и конкурсов для студентов.

- Scrum как гибкий управленческий фреймворк. Модель agileвзаимодействия субъектов в команде «университет - студент - работодатель» имеет гибкий управленческий контур. Постоянное взаимодействие субъектов в партнерском формате позволяет поддерживать актуальную адекватную картину о происходящем и своевременно реагировать на изменения.

2. Модель практико-ориентированного обучения на основе agileпринципов. Модель agile-взаимодействия субъектов в команде «университет студент - работодатель» с фокусом на формировании профессиональных, личностных и мотивационных компетенций, удовлетворяющих требованиям работодателя отражена на рисунке.

Формально можно разделить модель на 5 основных блоков: а) процесс обучения; б) преподаватели кафедры; в) кураторы предприятий; г) востребованный выпускник; д) мотивированный абитуриент. Рассмотрим каждый блок более подробно.

Прочесс обучения - ядро изменений, центральный элемент модели. Отправной точкой изменений явился переход в 2016 году на программу прикладного бакалавриата по профилю «Менеджмент» со значительной корректировкой учебных планов: увеличение практик в 6 раз, с 9 до 54 зачетных единиц. Дополнительно к учебной, производственной и преддипломной практике была введена семестровая практика по восьми профилирующим курсам. В результате студенты имеют возможность один день в неделю на протяжении всего семестра стажироваться на предприятии, знакомясь с производственными процессами и нарабатывая практические навыки решения различных задач. Именно в этом заключается практико-ориентированное обучение. С целью профессионального самоопределения на протяжении четырех лет обучения студенты имеют возможность пройти стажировку на предприятиях различных сфер деятельности. 


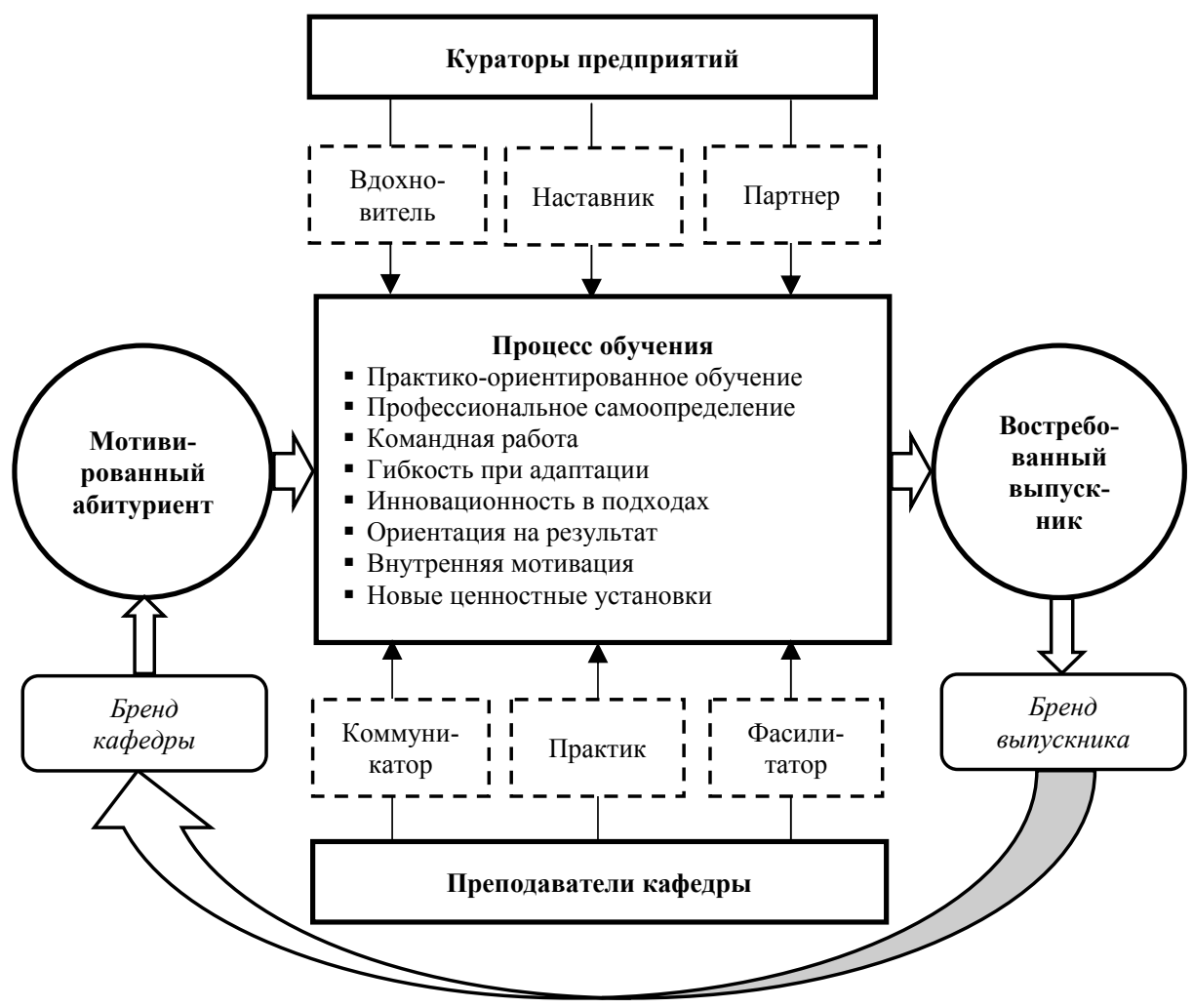

Рис. Модель практико-ориентированного обучения на основе agile-принципов (разработано авторами)

Командная работа как один из организационно-мотивационных механизмов модели позволяет достигать синергетического эффекта от взаимодействия субъектов модели «университет-студент-работодатель». Проактивное поведение позволяет сформировать самоорганизующуюся кросс-функциональную команду. Студент и работодатель из «пассивных» потребителей жизнедеятельности образовательной системы превращаются в активных субъектов, нацеленных на общий результат. Ключевая командная цель - формирование профессиональных, личностных и мотивационных компетенций студентов, отвечающих требованиям и вызовам современной окружающей среды.

Функционирование образовательной системы непрерывно сопровождается внутренними и внешними изменениями. Поэтому важным качеством системы или организационным инструментом является гибкость при адаптации.

Для поддержания высоких показателей по качеству образовательных услуг, а также для удержания хороших конкурентных позиций характерной чертой agile-модели является инноваџионность в подходах. Это проявляется в содержательных аспектах процесса обучения, в технологиях и форматах обучения, а также в поведенческих компетенциях субъектов модели. 
Ориентация на результат - по сравнению с традиционной системой высшего образования в agile-модели есть 2 отличия: 1) под результатом понимается не только конечный результат системы - выпускник, но и промежуточные результаты: студент какого-либо курса с определенным набором компетенций, который стажируется на предприятии; 2) восприятие результата каждым преподавателем, передающим знания и навыки, происходит в комплексной взаимосвязи с другими дисциплинами и в привязке не только к профессиональным, но и к личностно-мотивационным компетенциям.

Жизнеспособность и результативность agile-модели строится на внуmренней мотивации ее субъектов. Безусловно, у каждого субъекта своя индивидуальная внутренняя мотивация, но объединяющим является мотив достижения - стремление к востребованности и конкурентоспособности.

Новые ценностные установки являются фундаментом нового подхода к образовательному процессу: практико-ориентированность, адаптивность, гибкость, инновационность сменяют классические ценности университета академичность, устойчивость, фундаментальность.

Agile-модель меняет профиль преподавателя кафедры: как субъект системы дополнительно к базовой роли носителя знаний он выступает в новых ролях - коммуникатора, практика, фасилитатора.

Kураторы предприятий. Работодатель становится партнером системы. Активное взаимодействие студентов и работодателя, начиная с первого курca, позволяет постоянно актуализировать ожидания работодателей, корректировать способности и настрой студентов, корректировать содержание учебных курсов. В agile-модели у работодателя в лице кураторов появляется новый профиль-вдохновитель, наставник, партнер.

Востребованный выпускник - четвертый элемент agile-модели, продукт или результат образовательной системы, является индикатором качества работы модели и одновременно способом привлечения мотивированных абитуриентов за счет бренда кафедры как структурного подразделения университета.

Таким образом, agile-принципы, заложенные в модели прикладного бакалавриата, позволяют трансформировать традиционную систему обучения благодаря гибким организационно-мотивационным механизмам, новым ролям и ценностным установкам субъектов модели, что в совокупности создает саморазвиваюшуюся адаптивную систему выращчивания кадров под потребности предприятий.

3. Результаты внедрения модели практико-ориентированного обучения на основе agile-принципов. С момента своего внедрения за последние 1,5 года agileмодель практико-ориентированного обучения показала свою результативность. Значения показателей, наиболее ярко отражающих динамику изменений, приведены в таблице в формате «до внедрения» и «после внедрения» модели. Аналитика, отражающая эффективность процесса обучения до внедрения модели, 
представляет собой средние показатели за 2014-2015 годы. Сбор данных по индикаторам осуществляется методом обработки статистических данных, собираемых на кафедре в процессе обучения и методом анкетирования.

Значения показателей до и после внедрения модели

\begin{tabular}{|c|l|c|c|}
\hline $\begin{array}{c}\text { № } \\
\text { п/п }\end{array}$ & \multicolumn{1}{|c|}{ Индикаторы эффективности } & $\begin{array}{c}\text { До вне- } \\
\text { вне- } \\
\text { дрения } \\
\text { модели }\end{array}$ & $\begin{array}{c}\text { После } \\
\text { вндрения } \\
\text { модели }\end{array}$ \\
\hline 1 & $\begin{array}{l}\text { Доля студентов, успешно (4 или 5 баллов по 5-балльной } \\
\text { шкале) сдавших экзамены по профильным дисциплинам }\end{array}$ & $69 \%$ & $84 \%$ \\
\hline 2 & $\begin{array}{l}\text { Уровень удовлетворенности студентов учебным процессом } \\
\text { по 100-балльной шкале, замеряемой через анкетирование } \\
\text { в разрезе дисциплин }\end{array}$ & $62 \%$ & $79 \%$ \\
\hline 3 & $\begin{array}{l}\text { Количество предприятий (потенциальных работодателей) } \\
\text { в базе для устройства на практику }\end{array}$ & 7 \\
\hline 4 & $\begin{array}{l}\text { Доля студентов, которые в период обучения в университете } \\
\text { начинают работать по специальности }\end{array}$ & $12 \%$ \\
\hline 5 & $\begin{array}{l}\text { Количество работодателей в год, обращающихся с просьбой } \\
\text { подбора студента для трудоустройства }\end{array}$ & $32 \%$ \\
\hline 6 & $\begin{array}{l}\text { Доля студентов, привлекаемых для реализации коммерче- } \\
\text { ского проекта, заказчиком которого является предприятие - } \\
\text { потенциальный работодатель }\end{array}$ & $19 \%$ \\
\hline
\end{tabular}

В целом все индикаторы демонстрируют три классических ключевых положительных эффекта, получаемых от внедрения agile-технологий: повышение качества, ускорение прочессов, снижение затрат. Большинство индикаторов сочетают в себе сразу два или три положительных эффекта. Стоит отметить, что эффект, связанный со снижением затрат, имеет лишь косвенное проявление в приведенных индикаторах. Однако бесспорно положительный экономический эффект присутствует, когда мы видим: что выпускники, обладая компетенциями на должном уровне, не требуют существенных инвестиций от работодателя в процессы адаптации и повышения квалификация; что студенты, привлекаемые для реализации коммерческих проектов, приносят выгоду кафедре; что для привлечения абитуриентов не нужны большие вложения, так как привлекательный бренд кафедры и выпускника является лучшим маркетинговым средством продвижения образовательных услуг на рынке.

Таким образом, приведенные статистические данные с положительной динамикой доказывают состоятельность внедренной управленческой инновации и демонстрируют значительный прирост по параметрам качества и скорости подготовки студентов.

Заключение. Результаты внедрения agile-модели практико-ориентированного обучения студентов на кафедре менеджмента и маркетинга ПНИПУ позволяют выделить приобретаемые выгоды: трансформация модели управления существенно повышает качество менеджмента, обеспечивает возмож- 
ность интеграции с другими университетами и предприятиями в рамках реализации концепции университета национальной технологической инициативы 2035 [18], формирует преподавателей «нового» поколения, рост человеческого капитала является одной из ключевых задач в современной сфере образования. Изменения в формате взаимодействия «университет-студентработодатель» обеспечивают ускорение и опережающее предложение «готового» продукта с переносом фокуса образовательных программ с развития предметных знаний и запоминания информации на развитие личностных и метапредметных компетенций [1]. Безусловно, успешность инновационной управленческой технологии для университета может быть достигнута только при полной поддержке менеджмента и подготовки профессорско-преподавательского состава к поэтапной реализации гибких подходов [19].

\section{Список литературы}

1. The Boston Consulting Group. Россия 2025: от кадров к талантам [Электронный ресурс]. - M., 2017. - 72 c. - URL: http://d-russia.ru/wp-content/uploads/ 2017/11/Skills_Outline_web_tcm26-175469.pdf (дата обращения: 01.07. 2018).

2. De Smet A., Aghina W. The keys to organizational agility. - McKinsey\& Company, 2015. - URL: https://www.mckinsey.com/business-functions/organization/our-insights/the-keys-to-organizational-agility (accessed 01 July 2018).

3. Rigby D.K, Sutherland J., Takeuchi H. The Secret History of Agile Innovation // Harvard Business Review. - 2016. - № 4. - URL: https://hbr.org/ 2016/04/the-secret-history-of-agile-innovation (accessed 01 July 2018).

4. Denning S. Explaining Agile // Forbes. 8 September 2016. - URL: https://www.forbes.com/sites/stevedenning/2016/09/08/explaining-agile/\#2ffbae930 1 b8 (accessed 01 July 2018).

5. Agile Alliance [Электронный pecypc]. - URL: https://www.agilealliance.org/agile101/ (дата обращения: 01.07.2018). $144 \mathrm{c}$.

6. Вольфсон Б. Гибкие методологии разработки. - СПб.: Питер, 2017. -

7. Sutherland J. Scrum: The Art of Doing Twice the Work in Half the Time. Currency, 2014. -256 p.

8. Цирюльник С. Осторожно, Agile! Высокая вероятность подхватить Scrum головного мозга [Электронный ресурс]. - URL: https://medium.com/ @ neemah/осторожно-agile-высокая-вероятность-подхватить-scrum-головногомозга-a6febc94b453 (дата обращения: 01.07. 2018).

9. Šochová Z. The Great ScrumMaster: \#ScrumMasterWay. - AddisonWesley Professional, 2017. - 176 p.

10. The Agile Manifesto. - URL: http://agilemanifesto.org/iso/ru/manifesto.html (accessed 01 July 2018). 
11. Dingsøyr T., Nerur S., Balijepally V.G., Moe N.B. A decade of agile methodologies: Towards explaining agile software development // The Journal of Systems and Software. - 2012. - № 85 (6). - P. 1213-1221.

12. VersionOne. The 11 annual State of Agile report. 2017. - URL: http://www.agile247.pl/wp-content/uploads/2017/04/versionone-11th-annual-state-ofagile-report.pdf (accessed 01 July 2018).

13. Agile Project Manager Salaries [Электронный pecypc]. - URL: https://www.glassdoor.co.uk/ Salaries/agile-project-manager-salary-SRCH_KO0,21.htm (дата обращения: 01.07.2018).

14. Agile как педтехнология [Электронный ресурc]. - URL: http:// agileineducation.ru/category/agile-gde/agile-kak-pedtexnologiya/ (дата обращения: 01.07.2018).

15. Кузнецов Е. Университеты 3.0. в НТИ [Электронный ресурс]. URL: https://ioe.hse.ru/data/2016/01/13/1134679511/\%D0\%A3\%D0\%BD\%D0\%B 8\%D0\%B2\%D0\%B5\%D1\%80\%D1\%81\%D0\%B8\%D1\%82\%D0\%B5\%D1\%82\% D1\%8B\%203.0.pdf (дата обращения: 01.07.2018).

16. Применение методики EduScrum в образовании [Электронный реcypc]. - URL: http://eduscrum.nl/en/ (дата обращения: 01.07.2018).

17. Гульчевская H. Agile в проектной деятельности детей [Электронный pecypc]. - URL: http://agileineducation.ru/wp-content/uploads/2017/11/Agile-впроектной-деятельности-детей-22-11-17.pdf (дата обращения: 01.07.2018).

18. Иващенко А. Как нацелить науку на решение задач бизнеса [Электронный ресурс]. - URL: http://ntinews.ru/blog/publications/kak-natselit-naukuna-reshenie-zadach-biznesa.html (дата обращения: 01.07.2018).

19. Молодчик А.В. Современные механизмы развития инновационного потенциала экономики в системе отношений «вуз-предприятие» // Вестник Пермского национального исследовательского политехнического университета. Социально-экономические науки, 2017. - № 3. - С. 183-191.

20. Adkins L. Coaching Agile Teams: A Companion for ScrumMasters, Agile Coaches, and Project Managers in Transition. - Addison-Wesley Professional, 2010. $-352 \mathrm{p}$.

\section{References}

1. The Boston Consulting Group. Rossiia 2025: ot kadrov k talantam [Russia 2025: From personnel to talents]. Moscow, 2017, available at: http://drussia.ru/wp-content/uploads/2017/11/Skills_Outline_web_tcm26-175469.pdf (accessed 01 July 2018).

2. De Smet A., Aghina W. The keys to organizational agility. McKinsey\& Company, 2015, available at: https://www.mckinsey.com/business-functions/organization/our-insights/the-keys-to-organizational-agility (accessed 01 July 2018). 
3. Rigby D.K., Sutherland J., Takeuchi H. The secret history of agile innovation. Harvard Business Review, 2016, no. 4, available at: https://hbr.org/2016/04/ the-secret-history-of-agile-innovation (accessed 01 July 2018).

4. Denning S. Explaining Agile. Forbes. 08 September 2016, available at: https://www.forbes.com/sites/stevedenning/2016/09/08/explainingagile/\#2ffbae930 1b8 (accessed 01 July 2018).

5. Agile Alliance, available at: https://www.agilealliance.org/agile101/ (accessed 01 July 2018).

6. Vol'fson B. Gibkie metodologii razrabotki [Agile methodologies for the development]. St. Petersburg, Piter, 2017, 144 p.

7. Sutherland J. Scrum: The Art of doing twice the work in half the time. Currency, 2014, 256 p.

8. Tsiriul'nik S. Ostorozhno, Agile! Vysokaia veroiatnost' podkhvatit' Scrum golovnogo mozga [Be careful, Agile! High probability to catch up brain Scrum]. 2016, available at: https://medium.com/@neemah/осторожно-agile-высокаявероятность-подхватить-scrum-головного-мозга-a6febc94b453 (accessed 01 July 2018).

9. Šochová Z. The Great ScrumMaster: \#ScrumMasterWay. Addison-Wesley Professional, 2017, 176 p.

10. The Agile Manifesto, available at: http://agilemanifesto.org/ (accessed 01 July 2018).

11. Dingsøyr T., Nerur S., Balijepally V.G., Moe N.B. A decade of agile methodologies: Towards explaining agile software development. The Journal of Systems and Software, 2012, no. 85 (6), pp. 1213-1221.

12. VersionOne. The 11 annual State of Agile report, 2017, available at: http://www.agile247.pl/wp-content/uploads/2017/04/versionone-11th-annual-stateof-agile-report.pdf (accessed 01 July 2018).

13. Agile Project Manager Salaries, available at: https://www.glassdoor.co.uk/ Salaries/agile-project-manager-salary-SRCH_KO0,21.htm (accessed 01 July 2018).

14. Agile kak pedtekhnologiia [Agile as an education technology]. Available at: http://agileineducation.ru/category/agile-gde/agile-kak-pedtexnologiya/ (accessed 01 July 2018).

15. Kuznetsov E. Universitety 3.0. v NTI [University 3.0 in NTI]. Available at: $\quad$ https://ioe.hse.ru/data/2016/01/13/1134679511/\%D0\%A3\%D0\%BD\%D0\%B $8 \%$ D0\%B2\%D0\%B5\%D1\%80\%D1\%81\%D0\%B8\%D1\%82\%D0\%B5\%D1\%82\% D1\%8B\%203.0.pdf (accessed 01 July 2018).

16. EduScrum, available at: http://eduscrum.nl/en/ (accessed 01 July 2018).

17. Gul'chevskaia N. Agile v proektnoi deiatel'nosti detei [Agile in children project activity]. Available at: http://agileineducation.ru/wp-content/uploads/2017/ 11/Agile-в-проектной-деятельности-детей-22-11-17.pdf (accessed 01 July 2018).

18. Ivashchenko A. Kak natselit' nauku na reshenie zadach biznesa [How to focus the science on business issues]. Available at: http://ntinews.ru/blog/publications/kak-natselit-nauku-na-reshenie-zadach-biznesa.html (accessed 01 July 2018). 
19. Molodchik A.V. Sovremennye mekhanizmy razvitiia innovatsionnogo potentsiala ekonomiki v sisteme otnoshenii "vuz-predpriiatie" [Modern mechanisms of economy's innovative potential development in the system of relations between university and enterprise]. PNRPU Sociology and Economics Bulletin, 2017, no. 3, pp. 183-191.

20. Adkins L. Coaching Agile teams: A Companion for Scrum masters, Agile coaches, and project managers in transition. Addison-Wesley Professional, $2010,352 \mathrm{p}$.

Оригинальность $92 \%$

Получено 20.07.2018 Принято 20.08.2018 Опубликовано 03.04.2019

\author{
N.A. Molodchik, N.I. Nagibina
}

\title{
PRACTICE-ORIENTED LEARNING MODEL DEVELOPMENT AND IMPLEMENTATION AT THE UNIVERSITY BASED ON AGILE PRINCIPLES
}

\begin{abstract}
Socio-economic and political challenges of the global world bring corrections to the current needs of the labor market: the need to produce new innovative products determines the need to accelerate the training of specialists with relevant competences with a focus on cognitive skills instead of knowledge transfer. In the conditions of optimization of state educational institutions, timely transformation is the only way for the majority of Russian universities to survive. The hypothesis of the research is the applicability of agile-concept in the field of higher education in Russia: the use of agile-principles in the management of structural units of the university can have a significant positive impact on improving the efficiency of the educational process and increasing the competitiveness of the university. The proposed model of practice-oriented education, based on the agile principles borrowed from the field of information technology confirmed its relevance at the enterprises in various fields of activity.

The model based on the concept of Agile - a new cultural phenomenon, way of thinking, philosophy, transforms the format and nature of interaction between university, student, and employer directly in the learning process: each of the subjects is an active element of the system and at any time can initiate any proposals or changes. The model formally identifies five main blocks: a) the learning process, b) department professors, c) curators of enterprises, d) a graduate required, e) a motivated applicant. The model testing in the management of the university's structural unit - the Department of Management and Marketing, Perm National Research Polytechnic University - has yielded positive dynamics of changes in three classic key effects: quality improvement, process acceleration, and cost reduction.
\end{abstract}

Keywords: practice-oriented learning model, agile-principles, university, implementation.

Natalya A. Molodchik - Candidate of Economic Sciences, Associate Professor, Department of Management and Marketing, Perm National Research Polytechnic University, e-mail: namdom@yandex.ru.

Natalya I. Nagibina - Candidate of Economic Sciences, Associate Professor, Department of Management and Marketing, Perm National Research Polytechnic University, e-mail: nagibinan@list.ru.

Received 20.07.2018 Accepted 20.08.2018 Published 03.04.2019 